European Physical Journal C 73, 2506 (2013).

\title{
Some exact results on CP and CPT violations in a $C=-1$ entangled pseudoscalar neutral meson pair
}

\author{
$\mathrm{Yu} \mathrm{Sh} *$ \\ Center for Field Theory and Particle Physics, Department of Physics, Fudan University, Shanghai 200433, China
}

\begin{abstract}
We consider neutral pseudoscalar mesons in an entangled or Einstein-Podolsky-Rosen state with $C=-1$. Due to quantum entanglement and antisymmetry of this state, the rates of the joint decays of the meson pair display various interesting features, as is well known. As functions of CP and CPT violating parameters, here we obtain some exact results about the joint decay rates and their asymmetries for a given time difference defined for joint decays to flavor eigenstates, as well as those for joint decays to $\mathrm{CP}$ eigenstates. The entanglement allows a meaningful and useful definition of the transition amplitude from a CP eigenstate of a meson. These results yield useful information and criteria on $\mathrm{CP}$ and $\mathrm{CPT}$ violations.
\end{abstract}

PACS numbers: 14.40.-n, 03.65.Ud

\section{INTRODUCTION}

Pseudoscalar neutral mesons are ideal systems in studying CP violation, and in search for CPT violation, which is implied by the standard model extension [1]. Pseudoscalar neutral mesons in an entangled state have special properties due to quantum entanglement or Einstein-Podolsky-Rosen correlation [2 7]. Nowadays, such entangled meson pairs are routinely produced in $\phi$ or $B$ factories [8 11]. Hence it is interesting and important to explore the use of the entangled pairs in $\mathrm{CP}$ and $\mathrm{CPT}$ problems 8 -26].

Previous investigations often used some approximations up to the first order of the CP or CPT violating parameters. Reasonable as it is, it is of special value to have some exact results without approximations, since being exactly zero is qualitatively different from being approximately zero with higher orders of CP or CPT violating parameters neglected. Moreover, exact results are important in making comparisons between different approximations.

In this paper, we present some exact results on the use of the $C=-1$ entangled state of pseudoscalar mesons to examine $\mathrm{CP}$ and $\mathrm{CPT}$ violations or conservations. A recent calculation ignored direct $\mathrm{CP}$ violation and the violation of $\Delta \mathcal{F}=\Delta Q$ rule, where $\mathcal{F}$ is the flavor quantum number [26].

The rest of this paper is organized as the following. In Sec. III we review the single-particle bases, the time evolution of a single neutral meson as well as that of an entangled neutral meson pair with total $C=-1$. In Sec. IIII, we consider the decays of both mesons into flavor eigenstates, for which some results concerning CP and CPT are given in Sec. IV] In Sec. V we consider decays of both mesons into CP eigenstates, for which some results concerning CP and CPT are given in Sec VI. A summary is made in Sec. VII.

\section{A REVIEW OF SINGLE NEUTRAL MESON AND ENTANGLED NEUTRAL MESONS}

A neutral pseudoscalar meson $M^{0}$ and its antiparticle $\bar{M}^{0}$ are eigenstates of parity $P$ both with eigenvalue -1 , and of a characteristic flavor $\mathcal{F}$ with eigenvalues $\pm 1 . \mathcal{F}$ is strangeness for $K^{0}$ and $\bar{K}^{0}$, beauty for $B_{d}^{0}$ and $\bar{B}_{d}^{0}$, charm for $D^{0}$ and $\bar{D}^{0}$, and strangeness or beauty (with a minus sign) for $B_{s}^{0}$ and $\bar{B}_{s}^{0}$. With the phase convention $C\left|M^{0}\right\rangle=-\left|\bar{M}^{0}\right\rangle$ and $C\left|\bar{M}^{0}\right\rangle=-\left|M^{0}\right\rangle$, the eigenstates of $C P$ are

$$
\left|M_{ \pm}\right\rangle=\frac{1}{\sqrt{2}}\left(\left|M^{0}\right\rangle \pm\left|\bar{M}^{0}\right\rangle\right)
$$

with eigenvalues \pm 1 . It should be noted that physically a single particle cannot be in the state $\left|M_{ \pm}\right\rangle$, as $\mathrm{CP}$ is violated.

*Electronic address: yushi@fudan.edu.cn 
In the flavor basis, the effective mass matrix $H$ can be written as

$$
H=\left(\begin{array}{cc}
H_{00} & H_{0 \overline{0}} \\
H_{\overline{0} 0} & H_{\overline{0} \overline{0}}
\end{array}\right),
$$

where $H_{00} \equiv\left\langle M^{0}|H| M^{0}\right\rangle, H_{0 \overline{0}} \equiv\left\langle M^{0}|H| \bar{M}^{0}\right\rangle, H_{\overline{0} 0} \equiv\left\langle\bar{M}^{0}|H| M^{0}\right\rangle, H_{\overline{0} \overline{0}} \equiv\left\langle\bar{M}^{0}|H| \bar{M}^{0}\right\rangle$.

Indirect $\mathrm{T}$ violation and $\mathrm{CP}$ violation are characterized by a nonzero parameter $\epsilon_{M}$ defined through

$$
\frac{q}{p} \equiv \sqrt{\frac{H_{\overline{0} 0}}{H_{0 \overline{0}}}} \equiv \frac{1-\epsilon_{M}}{1+\epsilon_{M}},
$$

because if $\mathrm{CP}$ or $\mathrm{T}$ is conserved indirectly, then $\epsilon_{M}=0$.

Indirect $\mathrm{CPT}$ violation and $\mathrm{CP}$ violation are characterized by a nonzero parameter $\delta_{M}$ defined as [27]

$$
\delta_{M} \equiv \frac{H_{\overline{0} \overline{0}}-H_{00}}{\sqrt{H_{0 \overline{0}} H_{\overline{0} 0}}} \neq 0,
$$

because if CPT or CP is conserved indirectly, then $\delta_{M}=0$.

The eigenvalues of $H$ are

$$
\begin{aligned}
& \lambda_{S} \equiv m_{S}-i \Gamma_{S} / 2=H_{00}+\sqrt{H_{0 \overline{0}} H_{\overline{0} 0}}\left(\sqrt{1+\frac{\delta_{M}^{2}}{4}}+\frac{\delta_{M}}{2}\right), \\
& \lambda_{L} \equiv m_{L}-i \Gamma_{L} / 2=H_{\overline{0} \overline{0}}-\sqrt{H_{0 \overline{0}} H_{\overline{0} 0}}\left(\sqrt{1+\frac{\delta_{M}^{2}}{4}}+\frac{\delta_{M}}{2}\right),
\end{aligned}
$$

corresponding, respectively, to the eigenstates

$$
\begin{aligned}
& \left|M_{S}\right\rangle=\frac{1}{\sqrt{\left|p_{S}\right|^{2}+\left|q_{S}\right|^{2}}}\left(p_{S}\left|M^{0}\right\rangle+q_{S}\left|\bar{M}^{0}\right\rangle\right)=\frac{1}{\sqrt{1+\left|\epsilon_{S}\right|^{2}}}\left(\left|M_{+}\right\rangle+\epsilon_{S}\left|M_{-}\right\rangle\right), \\
& \left|M_{L}\right\rangle=\frac{1}{\sqrt{\left|p_{L}\right|^{2}+\left|q_{L}\right|^{2}}}\left(p_{L}\left|M^{0}\right\rangle-q_{L}\left|\bar{M}^{0}\right\rangle\right)=\frac{1}{\sqrt{1+\left|\epsilon_{L}\right|^{2}}}\left(\epsilon_{L}\left|M_{+}\right\rangle+\left|M_{-}\right\rangle\right),
\end{aligned}
$$

with

$$
\begin{aligned}
& x_{S} \equiv \frac{q_{S}}{p_{S}} \equiv \frac{1-\epsilon_{S}}{1+\epsilon_{S}}=\frac{q}{p}\left(\sqrt{1+\frac{\delta_{M}^{2}}{4}}+\frac{\delta_{M}}{2}\right), \\
& x_{L} \equiv \frac{q_{L}}{p_{L}} \equiv \frac{1-\epsilon_{L}}{1+\epsilon_{L}}=\frac{q}{p}\left(\sqrt{1+\frac{\delta_{M}^{2}}{4}}-\frac{\delta_{M}}{2}\right),
\end{aligned}
$$

If $\epsilon_{S}=0,\left|M_{S}\right\rangle=\left|M_{+}\right\rangle$, with $C P\left|M_{+}\right\rangle=\left|M_{+}\right\rangle$. If $\epsilon_{L}=0,\left|M_{L}\right\rangle=\left|M_{-}\right\rangle$, with $C P\left|M_{-}\right\rangle=-\left|M_{-}\right\rangle$. Conversely

$$
\begin{aligned}
\left|M_{+}\right\rangle & =\frac{1}{1-\epsilon_{S} \epsilon_{L}}\left(\sqrt{1+\left|\epsilon_{S}\right|^{2}}\left|M_{S}\right\rangle-\epsilon_{S} \sqrt{1+\left|\epsilon_{L}\right|^{2}}\left|M_{L}\right\rangle\right), \\
\left|M_{-}\right\rangle & =\frac{1}{1-\epsilon_{S} \epsilon_{L}}\left(\sqrt{1+\left|\epsilon_{L}\right|^{2}}\left|M_{L}\right\rangle-\epsilon_{L} \sqrt{1+\left|\epsilon_{S}\right|^{2}}\left|M_{S}\right\rangle\right) .
\end{aligned}
$$

We often have the definitions

$$
\begin{gathered}
\epsilon \equiv \frac{1}{2}\left(\epsilon_{S}+\epsilon_{L}\right), \\
\delta \equiv \frac{1}{2}\left(\epsilon_{S}-\epsilon_{L}\right) .
\end{gathered}
$$

Hence

$$
\epsilon=\frac{\epsilon_{M}}{1+\epsilon_{M}^{2}+\left(1-\epsilon_{M}^{2}\right) \sqrt{1+\frac{\delta_{M}^{2}}{4}}} \approx \frac{\epsilon_{M}}{2},
$$




$$
\delta=-\frac{\left(1-\epsilon_{M}^{2}\right) \delta_{M}}{1+\epsilon_{M}^{2}+\left(1-\epsilon_{M}^{2}\right) \sqrt{1+\frac{\delta_{M}^{2}}{4}}} \approx-\frac{\delta_{M}}{2} .
$$

Under the Wigner-Weisskopf approximation, the evolution of an arbitrary state of a pseudoscalar meson $|M(t)\rangle$, as a superposition of $\left|M^{0}\right\rangle$ and $\left|\bar{M}^{0}\right\rangle$, can be described by a Schrödinger equation

$$
i \frac{\partial}{\partial t}|M(t)\rangle=H|M(t)\rangle
$$

using which we can find the following time-dependent states.

Starting as the mass eigenstate $\left|M_{S}\right\rangle$, the state of a single meson evolves as

$$
\left|M_{S}(t)\right\rangle=e^{-i \lambda_{S} t}\left|M_{S}\right\rangle
$$

Starting as the mass eigenstate $\left|M_{L}\right\rangle$, the state evolves as

$$
\left|M_{L}(t)\right\rangle=e^{-i \lambda_{L} t}\left|M_{L}\right\rangle
$$

Starting as the flavor eigenstate $\left|M^{0}\right\rangle$, the state evolves as

$$
\left|M^{0}(t)\right\rangle=G_{00}(t)\left|M^{0}\right\rangle+G_{0 \overline{0}}(t)\left|\bar{M}^{0}\right\rangle,
$$

where

$$
\begin{aligned}
G_{00}(t) & \equiv \frac{e^{-i \lambda_{S} t}+\Omega e^{-i \lambda_{L} t}}{1+\Omega}, \\
G_{0 \overline{0}}(t) & \equiv \frac{x_{S}\left(e^{-i \lambda_{S} t}-e^{-i \lambda_{L} t}\right)}{1+\Omega},
\end{aligned}
$$

with

$$
\Omega \equiv \frac{x_{S}}{x_{L}}=\frac{q_{S} p_{L}}{p_{S} q_{L}}
$$

Starting as the flavor eigenstate $\left|\bar{M}_{0}\right\rangle$, the state evolves as

$$
\left|\bar{M}^{0}(t)\right\rangle=G_{\overline{0} 0}(t)\left|M^{0}\right\rangle+G_{\overline{0} \overline{0}}(t)\left|\bar{M}^{0}\right\rangle,
$$

where

$$
\begin{aligned}
G_{\overline{0} 0}(t) & \equiv \frac{x_{S}^{-1}\left(e^{-i \lambda_{S} t}-e^{-i \lambda_{L} t}\right)}{1+\Omega^{-1}} \\
G_{\overline{0} \overline{0}}(t) & \equiv \frac{e^{-i \lambda_{S} t}+\Omega^{-1} e^{-i \lambda_{L} t}}{1+\Omega^{-1}}
\end{aligned}
$$

Physically an isolated single meson state cannot start as a CP eigenstate $\left|M_{ \pm}\right\rangle$, as $\mathrm{CP}$ is indeed violated. There is no way of tagging one meson to be in such an eigenstate by measuring the other if the two are prepared as an entangled pair. However, as explained below, the initial state of a pair of entangled mesons with $C=-1$ is exactly a superposition of two terms, in each of which the two mesons are in different CP eigenstates. Therefore it is useful to consider the evolution of the state of a meson starting as a $\mathrm{CP}$ eigenstate. Starting as the $\mathrm{CP}$ eigenstate $\left|M_{+}\right\rangle$, the state evolves as

$$
\left|M_{+}(t)\right\rangle=F_{++}(t)\left|M_{+}\right\rangle+F_{+-}(t)\left|M_{-}\right\rangle .
$$

where

$$
\begin{aligned}
& F_{++}(t)=\frac{1}{2(1+\Omega)}\left[\left(1+x_{L}^{-1}+x_{S}+\Omega\right) e^{-i \lambda_{S} t}+\left(1-x_{L}^{-1}-x_{S}+\Omega\right) e^{-i \lambda_{L} t}\right] \\
& F_{+-}(t)=\frac{1+x_{L}^{-1}-x_{S}-\Omega}{2(1+\Omega)}\left(e^{-i \lambda_{S} t}-e^{-i \lambda_{L} t}\right) .
\end{aligned}
$$


Starting as the CP eigenstate $\left|M_{-}\right\rangle$, the state evolves as

$$
\left|M_{-}(t)\right\rangle=F_{-+}(t)\left|M_{+}\right\rangle+F_{--}(t)\left|M_{-}\right\rangle .
$$

where

$$
\begin{aligned}
& F_{-+}(t)=\frac{1-x_{L}^{-1}+x_{S}-\Omega}{2(1+\Omega)}\left(e^{-i \lambda_{S} t}-e^{-i \lambda_{L} t}\right), \\
& F_{--}(t)=\frac{1}{2(1+\Omega)}\left[\left(1-x_{L}^{-1}-x_{S}+\Omega\right) e^{-i \lambda_{S} t}+\left(1+x_{L}^{-1}+x_{S}+\Omega\right) e^{-i \lambda_{L} t}\right] .
\end{aligned}
$$

Now consider the $C=-1$ entangled state of a pair of pseudoscalar mesons, which may be referred to as Alice (a) and Bob (b),

$$
\left|\Psi_{-}\right\rangle=\frac{1}{\sqrt{2}}\left(\left|M^{0}\right\rangle_{a}\left|\bar{M}^{0}\right\rangle_{b}-\left|\bar{M}^{0}\right\rangle_{a}\left|M^{0}\right\rangle_{b}\right)
$$

which can be produced from a source of $J^{P C}=1^{--}$.

A remarkable feature of $\left|\Psi_{-}\right\rangle$is that it is also exactly

$$
\left|\Psi_{-}\right\rangle=\frac{1}{\sqrt{2}}\left(\left|M_{-}\right\rangle_{a}\left|M_{+}\right\rangle_{b}-\left|M_{+}\right\rangle_{a}\left|M_{-}\right\rangle_{b}\right)
$$

This means that although a single meson cannot be in the state $\left|M_{ \pm}\right\rangle$, an entangled pair of mesons with $C=-1$ is exactly in the state $\frac{1}{\sqrt{2}}\left(\left|M_{-}\right\rangle_{a}\left|M_{+}\right\rangle_{b}-\left|M_{+}\right\rangle_{a}\left|M_{-}\right\rangle_{b}\right)$. There is no tagging here for the initial mesons, as only the final decay products are measured. This allows the use of (27) and (30) for the entangled pair, because of the linearity of quantum evolution and that the two entangled but separated mesons are non-interacting.

Starting as $\left|\Psi_{-}\right\rangle$, the state of the entangled meson pair evolves and decays to or produces certain products at $t_{a}$ and $t_{b}$, which may or may not be equal. To account for this situation, one has

$$
\begin{aligned}
\left|\Psi_{-}\left(t_{a}, t_{b}\right)\right\rangle & =\frac{1}{\sqrt{2}}\left(\left|M^{0}\left(t_{a}\right)\right\rangle_{a}\left|\bar{M}^{0}\left(t_{b}\right)\right\rangle_{b}-\left|\bar{M}^{0}\left(t_{a}\right)\right\rangle_{a}\left|M^{0}\left(t_{b}\right)\right\rangle_{b}\right) \\
& =\frac{1}{\sqrt{2}}\left(\left|M_{-}\left(t_{a}\right)\right\rangle_{a}\left|M_{+}\left(t_{b}\right)\right\rangle_{b}-\left|M_{+}\left(t_{a}\right)\right\rangle_{a}\left|M_{-}\left(t_{b}\right)\right\rangle_{b}\right) .
\end{aligned}
$$

The joint rate that Alice decays to $\left|\psi_{a}\right\rangle$ at $t_{a}$ while Bob decays to $\left|\psi_{b}\right\rangle$ at $t_{b}$ is

$$
I\left(\psi_{a}, t_{a} ; \psi_{b}, t_{b}\right)=\left|\left\langle\psi_{a}, \psi_{b}\left|\mathcal{H}_{a} \mathcal{H}_{b}\right| \Psi_{-}\left(t_{a}, t_{b}\right)\right\rangle\right|^{2},
$$

where $\mathcal{H}_{a}$ and $\mathcal{H}_{b}$ represent the Hamiltonians governing the decays of $a$ and $b$, respectively,

$$
\begin{aligned}
\left\langle\psi_{a}, \psi_{b}\left|\mathcal{H}_{a} \mathcal{H}_{b}\right| \Psi_{-}\left(t_{a}, t_{b}\right)\right\rangle & =\frac{1}{\sqrt{2}}\left(\left\langle\psi_{a}\left|\mathcal{H}_{a}\right| M^{0}\left(t_{a}\right)\right\rangle_{a}\left\langle\psi_{b}\left|\mathcal{H}_{b}\right| \bar{M}_{0}\left(t_{b}\right)\right\rangle_{b}-\left\langle\psi_{a}\left|\mathcal{H}_{a}\right| \bar{M}_{0}\left(t_{a}\right)\right\rangle_{a}\left\langle\psi_{b}\left|\mathcal{H}_{b}\right| M^{0}\left(t_{b}\right)\right\rangle_{b}\right) \\
& =\frac{1}{\sqrt{2}}\left(\left\langle\psi_{a}\left|\mathcal{H}_{a}\right| M_{-}\left(t_{a}\right)\right\rangle_{a}\left\langle\psi_{b}\left|\mathcal{H}_{b}\right| M_{+}\left(t_{b}\right)\right\rangle_{b}-\left\langle\psi_{a}\left|\mathcal{H}_{a}\right| M_{+}\left(t_{a}\right)\right\rangle_{a}\left\langle\psi_{b}\left|\mathcal{H}_{b}\right| M_{-}\left(t_{b}\right)\right\rangle_{b}\right)
\end{aligned}
$$

\section{DECAYS INTO FLAVOR EIGENSTATES}

Suppose from the entangled state $\left|\Psi_{-}\right\rangle$, Alice and Bob each decays or transits to a two-valued flavor eigenstate. Let us generically denote the flavor eigenstates as $\left|l^{ \pm}\right\rangle$, with eigenvalue \pm 1 , respectively. Examples for $\left|l^{+}\right\rangle$include the semileptonic decay products $M^{-} \bar{l} \nu, D^{-} D_{S}^{+}, D^{-} K^{+}, \pi^{-} D_{S}^{+}, \pi^{-} K^{+}$from $M^{0}=B^{0}$, and $D_{S}^{-} \pi^{+}, D_{S}^{-} D^{+}, K^{-} \pi^{+}$, $K^{-} D^{+}$from $M^{0}=B_{S}^{0}$. Examples for $\left|l^{-}\right\rangle$include the semileptonic decay products $M^{+} l \bar{\nu}, D^{+} D_{S}^{-}, D^{+} K^{-}, \pi^{+} D_{S}^{-}$, $\pi^{+} K^{-}$from $\bar{M}^{0}=B^{0}$, and $D_{S}^{+} \pi^{-}, D_{S}^{+} D^{-}, K^{+} \pi^{-}, K^{+} D^{-}$from $M^{0}=B_{S}^{0}$. In the CPLEAR experiment on kaons [5], $\left|l^{+}\right\rangle$and $\left|l^{-}\right\rangle$are products produced via interaction with bound nucleons.

One can calculate the amplitude of such a joint decay in which Alice decays to $\left|l_{a}^{x}\right\rangle$ at $t_{a}$ while Bob decays to $\left|l_{b}^{y}\right\rangle$ at $t_{b}$, where $x$ and $y$ each represents \pm 1 ,

$$
\begin{aligned}
\left\langle l_{a}^{x}, l_{b}^{y}\left|\mathcal{H}_{a} \mathcal{H}_{b}\right| \Psi_{-}\left(t_{a}, t_{b}\right)\right\rangle & =\frac{1}{\sqrt{2}}\left(\left\langle l_{a}^{x}\left|\mathcal{H}_{a}\right| M^{0}\left(t_{a}\right)\right\rangle_{a}\left\langle l_{b}^{y}\left|\mathcal{H}_{b}\right| \bar{M}^{0}\left(t_{b}\right)\right\rangle_{b}-\left\langle l_{a}^{x}\left|\mathcal{H}_{a}\right| \bar{M}^{0}\left(t_{a}\right)\right\rangle_{a}\left\langle l_{b}^{y}\left|\mathcal{H}_{b}\right| M^{0}\left(t_{b}\right)\right\rangle_{b}\right) \\
& =C\left(l_{a}^{x}, l_{b}^{y}\right) e^{-i\left(\lambda_{S} t_{a}+\lambda_{L} t_{b}\right)}+D\left(l_{a}^{x}, l_{b}^{y}\right) e^{-i\left(\lambda_{L} t_{a}+\lambda_{S} t_{b}\right)},
\end{aligned}
$$


with

$$
\begin{aligned}
C\left(l_{a}^{x}, l_{b}^{y}\right) & \equiv \frac{1}{\sqrt{2}(1+\Omega)}\left(-x_{L}^{-1} r_{a}^{x} r_{b}^{y}+r_{a}^{x} \bar{r}_{b}^{y}-\Omega \bar{r}_{a}^{x} r_{b}^{y}+x_{S} \bar{r}_{a}^{x} \bar{r}_{b}^{y}\right) \\
D\left(l_{a}^{x}, l_{b}^{y}\right) & \equiv \frac{1}{\sqrt{2}(1+\Omega)}\left(x_{L}^{-1} r_{a}^{x} r_{b}^{y}+\Omega r_{a}^{x} \bar{r}_{b}^{y}-\bar{r}_{a}^{x} r_{b}^{y}-x_{S} \bar{r}_{a}^{x} \bar{r}_{b}^{y}\right)
\end{aligned}
$$

where

$$
\begin{aligned}
r_{\alpha}^{x} & \equiv\left\langle l_{\alpha}^{x}\left|\mathcal{H}_{\alpha}\right| M^{0}\right\rangle_{\alpha} \\
\bar{r}_{\alpha}^{x} & \equiv\left\langle l_{\alpha}^{x}\left|\mathcal{H}_{\alpha}\right| \bar{M}_{0}\right\rangle_{\alpha}
\end{aligned}
$$

$\alpha=a, b$.

Therefore we obtain the joint rate

$$
\begin{aligned}
I\left(l_{a}^{x}, t_{a} ; l_{b}^{y}, t_{b}\right)= & e^{-\left(\Gamma_{S}+\Gamma_{L}\right) t_{a}}\left\{|C|^{2} e^{-\Gamma_{L} \Delta t}+|D|^{2} e^{-\Gamma_{S} \Delta t}\right. \\
& \left.+2 e^{-\frac{\Gamma_{S}+\Gamma_{L}}{2} \Delta t}\left[\Re\left(C^{*} D\right) \cos (\Delta m \Delta t)+\Im\left(C^{*} D\right) \sin (\Delta m \Delta t)\right]\right\},
\end{aligned}
$$

where $C \equiv C\left(l_{a}^{x}, l_{b}^{y}\right), D \equiv D\left(l_{a}^{x}, l_{b}^{y}\right)$.

In experiments, it is more convenient to use the integrated rate, herein defined as

$$
I^{\prime}\left(l_{a}^{x}, l_{b}^{y}, \Delta t\right)=\int_{0}^{\infty} I\left(l_{a}^{x}, t_{a} ; l_{b}^{y}, t_{a}+\Delta t\right) d t_{a},
$$

which is simply given by $I\left(l_{a}^{x}, t_{a} ; l_{b}^{y}, t_{a}+\Delta t\right)$ as in $\left(\underline{58)}\right.$, with $e^{-\left(\Gamma_{S}+\Gamma_{L}\right) t_{a}}$ replaced as $1 /\left(\Gamma_{S}+\Gamma_{L}\right)$.

We focus on the situation that $\left|l_{a}^{+}\right\rangle=\left|l_{b}^{+}\right\rangle=\left|l^{+}\right\rangle$and $\left|l_{a}^{-}\right\rangle=\left|l_{b}^{-}\right\rangle=\left|l^{-}\right\rangle$, hence

$$
\begin{aligned}
& r_{a}^{+}=r_{b}^{+} \equiv R^{+}=a+b, \\
& \bar{r}_{a}^{+}=\bar{r}_{b}^{+} \equiv S^{+}=c^{*}-d^{*}, \\
& r_{a}^{-}=r_{b}^{-} \equiv S^{-}=c+d, \\
& \bar{r}_{a}^{-}=\bar{r}_{b}^{-} \equiv R^{-}=a^{*}-b^{*},
\end{aligned}
$$

where the quantities $a, b, c$ and $d$ are the ones usually defined in literature [8, 28].

If CP is conserved directly, then we have $R^{+}=R^{-}$and $S^{+}=S^{-}$. If CPT is conserved directly, then we have $\left(R^{+}\right)^{*}=R^{-}$and $\left(S^{+}\right)^{*}=S^{-}$. If $\Delta \mathcal{F}=\Delta Q$ rule is respected, then we have $S^{ \pm}=0$.

One obtains

$$
\begin{aligned}
& C\left(l^{+}, l^{+}\right)=-D\left(l^{+}, l^{+}\right)=\frac{1}{\sqrt{2}(1+\Omega)}\left[-x_{L}^{-1}\left(R^{+}\right)^{2}+(1-\Omega) R^{+} S^{+}+x_{S}\left(S^{+}\right)^{2}\right], \\
& C\left(l^{-}, l^{-}\right)=-D\left(l^{+}, l^{+}\right)=\frac{1}{\sqrt{2}(1+\Omega)}\left[-x_{L}^{-1}\left(S^{-}\right)^{2}+(1-\Omega) R^{-} S^{-}+x_{S}\left(R^{-}\right)^{2}\right], \\
& C\left(l^{+}, l^{-}\right)=-D\left(l^{-}, l^{+}\right)=U \\
& D\left(l^{+}, l^{-}\right)=-C\left(l^{-}, l^{+}\right)=V,
\end{aligned}
$$

where

$$
\begin{gathered}
U \equiv \frac{1}{\sqrt{2}(1+\Omega)}\left[-x_{L}^{-1} R^{+} S^{-}+x_{S} S^{+} R^{-}+R^{+} R^{-}-\Omega S^{+} S^{-}\right] \\
V \equiv \frac{1}{\sqrt{2}(1+\Omega)}\left[x_{L}^{-1} R^{+} S^{-}-x_{S} S^{+} R^{-}+\Omega R^{+} R^{-}-S^{+} S^{-}\right] .
\end{gathered}
$$

Therefore we obtain

$$
\begin{aligned}
& I\left(l^{+}, t_{a} ; l^{+}, t_{b}\right)=\frac{\left|-x_{L}^{-1}\left(R^{+}\right)^{2}+(1-\Omega) R^{+} S^{+}+x_{S}\left(S^{+}\right)^{2}\right|^{2}}{2|1+\Omega|^{2}} e^{-\left(\Gamma_{S}+\Gamma_{L}\right) t_{a}}\left[e^{-\Gamma_{L} \Delta t}+e^{-\Gamma_{S} \Delta t}-2 e^{-\frac{\Gamma_{S}+\Gamma_{L}}{2} \Delta t} \cos (\Delta m \Delta t)\right], \\
& I\left(l^{-}, t_{a} ; l^{-}, t_{b}\right)=\frac{\left|-x_{L}^{-1}\left(S^{-}\right)^{2}+(1-\Omega) R^{-} S^{-}+x_{S}\left(R^{-}\right)^{2}\right|^{2}}{2|1+\Omega|^{2}} e^{-\left(\Gamma_{S}+\Gamma_{L}\right) t_{a}}\left[e^{-\Gamma_{L} \Delta t}+e^{-\Gamma_{S} \Delta t}-2 e^{-\frac{\Gamma_{S}+\Gamma_{L}}{2} \Delta t} \cos (\Delta m \Delta t)\right], \\
& I\left(l^{+}, t_{a} ; l^{-}, t_{b}\right)=e^{-\left(\Gamma_{S}+\Gamma_{L}\right) t_{a}}\left\{|U|^{2} e^{-\Gamma_{L} \Delta t}+|V|^{2} e^{-\Gamma_{S} \Delta t}+2 e^{-\frac{\Gamma_{S}+\Gamma_{L}}{2} \Delta t}\left[\Re\left(U^{*} V\right) \cos (\Delta m \Delta t)+\Im\left(U^{*} V\right) \sin (\Delta m \Delta t)\right]\right\}, \\
& I\left(l^{-}, t_{a} ; l^{+}, t_{b}\right)=e^{-\left(\Gamma_{S}+\Gamma_{L}\right) t_{a}}\left\{|V|^{2} e^{-\Gamma_{L} \Delta t}+|U|^{2} e^{-\Gamma_{S} \Delta t}+2 e^{-\frac{\Gamma_{S}+\Gamma_{L}}{2} \Delta t}\left[\Re\left(U^{*} V\right) \cos (\Delta m \Delta t)-\Im\left(U^{*} V\right) \sin (\Delta m \Delta t)\right]\right\},
\end{aligned}
$$

where $\Delta m \equiv m_{L}-m_{S}, \Delta t \equiv t_{b}-t_{a}$. 


\section{CP AND CPT VIOLATIONS IN JOINT DECAYS INTO FLAVOR EIGENSTATES}

The above four joint rates can form some asymmetries among them. As $I^{\prime}\left(l^{x}, l^{y}, \Delta t\right) e^{-\left(\Gamma_{L}+\Gamma_{S}\right) t_{a}}=$ $\frac{1}{\Gamma_{L}+\Gamma_{S}} I\left(l^{x}, t_{a} ; l^{y}, t_{b}\right)$, the asymmetries defined for the instantaneous joint rate $I\left(l^{x}, t_{a} ; l^{y}, t_{a}+\Delta t\right)$ and its integration $I^{\prime}\left(l^{x}, l^{y}, \Delta t\right)$ for a specific $\Delta t$ are equal, and depend only on $\Delta t$,

$$
A\left(l^{x} l^{y}, l^{z} l^{w}, \Delta t\right) \equiv \frac{I\left[l^{x}, t_{a} ; l^{y}, t_{a}+\Delta t\right]-I\left[l^{z}, t_{a} ; l^{w}, t_{a}+\Delta t\right]}{I\left[l^{x}, t_{a} ; l^{y}, t_{a}+\Delta t\right]+I\left[l^{z}, t_{a} ; l^{w}, t_{a}+\Delta t\right]}=\frac{I^{\prime}\left[l^{x}, l^{y}, \Delta t\right]-I^{\prime}\left[l^{z}, l^{w}, \Delta t\right]}{I^{\prime}\left[l^{x}, l^{y}, \Delta t\right]+I^{\prime}\left[l^{z}, l^{w}, \Delta t\right]} .
$$

\section{A. Equal-flavor asymmetry}

Now we consider the equal-flavor asymmetry, which is

$$
A(++,--, \Delta t)=\frac{\left|x_{S}\left(S^{+}\right)^{2}-x_{L}^{-1}\left(R^{+}\right)^{2}+(1-\Omega) R^{+} S^{+}\right|^{2}-\left|x_{S}\left(R^{-}\right)^{2}-x_{L}^{-1}\left(S^{-}\right)^{2}+(1-\Omega) R^{-} S^{-}\right|^{2}}{\left|x_{S}\left(S^{+}\right)^{2}-x_{L}^{-1}\left(R^{+}\right)^{2}+(1-\Omega) R^{+} S^{+}\right|^{2}+\left|x_{S}\left(R^{-}\right)^{2}-x_{L}^{-1}\left(S^{-}\right)^{2}+(1-\Omega) R^{-} S^{-}\right|^{2}},
$$

which, because of the antisymmetry of $\left|\Psi_{-}\right\rangle$, is analogous to the famous Kabir asymmetry defined for the difference between the transition rates from $M^{0}$ to $\bar{M}^{0}$ and that from $\bar{M}^{0}$ to $M^{0}$ [22]. Note that $A(++,--, \Delta t)$ is a constant independent of $\Delta t$.

If CP is conserved directly, then $R^{+}=R^{-}, S^{+}=S^{-}$, consequently

$$
A(++,--, \Delta t)=\frac{\left|x_{S}\left(\zeta_{+}\right)^{2}-x_{L}^{-1}+(1-\Omega) \zeta_{+}\right|^{2}-\left|x_{S}-x_{L}^{-1}\left(\zeta_{+}\right)^{2}+(1-\Omega) \zeta_{+}\right|^{2}}{\left|x_{S}\left(\zeta_{+}\right)^{2}-x_{L}^{-1}+(1-\Omega) \zeta_{+}\right|^{2}-\left|x_{S}-x_{L}^{-1}\left(\zeta_{+}\right)^{2}+(1-\Omega) \zeta_{+}\right|^{2}}
$$

where

$$
\zeta_{ \pm} \equiv \frac{S^{ \pm}}{R^{ \pm}}
$$

which characterizes the violation of $\Delta \mathcal{F}=\Delta Q$ rule.

If CPT is conserved directly, then $\left(R^{+}\right)^{*}=R^{-}$and $\left(S^{+}\right)^{*}=S^{-}$, consequently

$$
A(++,--, \Delta t)=\frac{\left|x_{S}\left(\zeta_{+}\right)^{2}-x_{L}^{-1}+(1-\Omega) \zeta_{+}\right|^{2}-\left|x_{S}^{*}-\left(x_{L}^{*}\right)^{-1}\left(\zeta_{+}\right)^{2}+\left(1-\Omega^{*}\right) \zeta_{+}\right|^{2}}{\left|x_{S}\left(\zeta_{+}\right)^{2}-x_{L}^{-1}+(1-\Omega) \zeta_{+}\right|^{2}+\left|x_{S}^{*}-\left(x_{L}^{*}\right)^{-1}\left(\zeta_{+}\right)^{2}+\left(1-\Omega^{*}\right) \zeta_{+}\right|^{2}} .
$$

We can now obtain $A(++,--, \Delta t)$ for various combination cases.

If both CP and CPT are conserved directly, then $R^{+}=R^{-}$and $S^{+}=S^{-}$are both real numbers, consequently $A(++,--, \Delta t)$ is still given by (61), now with $\zeta_{+}$being real number.

If $\mathrm{CP}$ is conserved indirectly, no matter whether $\mathrm{CPT}$ is conserved indirectly, then $\epsilon_{M}=\delta_{M}=0$ and thus $x_{S}=x_{L}=\Omega=1$, consequently

$$
A(++,--, \Delta t)=\frac{\left|\left(S^{+}\right)^{2}-\left(R^{+}\right)^{2}\right|^{2}-\left|\left(R^{-}\right)^{2}-\left(S^{-}\right)^{2}\right|^{2}}{\left|\left(S^{+}\right)^{2}-\left(R^{+}\right)^{2}\right|^{2}+\left|\left(R^{-}\right)^{2}-\left(S^{-}\right)^{2}\right|^{2}}
$$

If CPT is conserved indirectly, then $\delta_{M}=0$, thus $x_{S}=x_{L}=q / p$, and thus $\Omega=1$, consequently

$$
A(++,--, \Delta t)=\frac{\left|\frac{q}{p}\left(S^{+}\right)^{2}-\frac{p}{q}\left(R^{+}\right)^{2}\right|^{2}-\left|\frac{q}{p}\left(R^{-}\right)^{2}-\frac{p}{q}\left(S^{-}\right)^{2}\right|^{2}}{\left|\frac{q}{p}\left(S^{+}\right)^{2}-\frac{p}{q}\left(R^{+}\right)^{2}\right|^{2}+\left|\frac{q}{p}\left(R^{-}\right)^{2}-\frac{p}{q}\left(S^{-}\right)^{2}\right|^{2}} .
$$

If $\mathrm{CP}$ is conserved both directly and indirectly, then

$$
A(++,--, \Delta t)=0 .
$$

If CPT is conserved both directly and indirectly, then

$$
\begin{aligned}
A(++,--, \Delta t) & =\frac{\left|\frac{q}{p}\left(\zeta_{+}\right)^{2}-\frac{p}{q}\right|^{2}-\left|\frac{q^{*}}{p^{*}}-\frac{p^{*}}{q^{*}}\left(\zeta_{+}\right)^{2}\right|^{2}}{\left|\frac{q}{p}\left(\zeta_{+}\right)^{2}-\frac{p}{q}\right|^{2}+\left|\frac{q^{*}}{p^{*}}-\frac{p^{*}}{q^{*}}\left(\zeta_{+}\right)^{2}\right|^{2}} \\
& =\frac{\left(\left|\frac{q}{p}\right|^{2}-\left|\frac{p}{q}\right|^{2}\right)\left(\left|\zeta_{+}\right|^{4}-1\right)}{\left(\left|\frac{q}{p}\right|^{2}+\left|\frac{p}{q}\right|^{2}\right)\left(\left|\zeta_{+}\right|^{4}+1\right)-4 \Re\left(\frac{q p^{*}}{p q^{*}}\left(\zeta_{+}\right)^{2}\right)} .
\end{aligned}
$$


If $\mathrm{CP}$ is conserved directly while CPT is conserved indirectly, then

$$
\begin{aligned}
A(++,--, \Delta t) & =\frac{\left|\frac{q}{p}\left(\zeta_{+}\right)^{2}-\frac{p}{q}\right|^{2}-\left|\frac{q}{p}-\frac{p}{q}\left(\zeta_{+}\right)^{2}\right|^{2}}{\left|\frac{q}{p}\left(\zeta_{+}\right)^{2}-\frac{p}{q}\right|^{2}+\left|\frac{q}{p}-\frac{p}{q}\left(\zeta_{+}\right)^{2}\right|^{2}} \\
& =\frac{\left(\left|\frac{q}{p}\right|^{2}-\left|\frac{p}{q}\right|^{2}\right)\left(\left|\zeta_{+}\right|^{4}-1\right)-4 \Im\left(\frac{q p^{*}}{p q^{*}}\right) \Im\left(\left(\zeta_{+}\right)^{2}\right)}{\left(\left|\frac{q}{p}\right|^{2}+\left|\frac{p}{q}\right|^{2}\right)\left(\left|\zeta_{+}\right|^{4}+1\right)-4 \Re\left(\frac{q p^{*}}{p q^{*}}\right) \Re\left(\left(\zeta_{+}\right)^{2}\right)} .
\end{aligned}
$$

If $\mathrm{CP}$ is conserved indirectly while CPT is conserved directly, then

$$
A(++,--, \Delta t)=0
$$

Combining the above two cases of $A(++,--, \Delta t)=0$, as stated in (66) and (71), we obtain the following exact theorems.

Theorem 1 If the equal-flavor asymmetry $A(++,--, \Delta t) \neq 0$, then we have one or two of the following violations: (1) CP is violated indirectly, (2) both CP and CPT are violated directly. Therefore assuming direct CPT conservation, nonzero $A(++,--, \Delta t)$ implies that $C P$ must be violated indirectly.

Moreover, under the assumption that CPT is conserved both directly and indirectly, if $A(++,--, \Delta t) \neq 0$, then both factors in the numerator in (68) must be nonzero. Hence we have the following exact theorem, which is clearly consistent with Theorem 1 , and is especially useful in testing $\mathrm{T}$ violation.

Theorem 2 If the equal-flavor asymmetry $A(++,--, \Delta t) \neq 0$ while CPT is assumed to be conserved both directly and indirectly, then in addition to indirect $C P$ violation, we can draw the following conclusions: (1) $|q / p| \neq 1$, i.e. $T$ must also be violated indirectly; (2) $\left|\zeta_{+}\right| \neq 1$, i.e. $\left|\left\langle l^{+}|\mathcal{H}| \bar{M}_{0}\right\rangle\right| \neq\left|\left\langle l^{+}|\mathcal{H}| M^{0}\right\rangle\right|,\left|\left\langle l^{-}|\mathcal{H}| M^{0}\right\rangle\right| \neq\left|\left\langle l^{-}|\mathcal{H}| \bar{M}_{0}\right\rangle\right|$, despite $\left\langle l^{+}|\mathcal{H}| M^{0}\right\rangle=\left\langle l^{-}|\mathcal{H}| \bar{M}_{0}\right\rangle^{*}$ and $\left\langle l^{+}|\mathcal{H}| \bar{M}_{0}\right\rangle=\left\langle l^{-}|\mathcal{H}| M^{0}\right\rangle^{*}$.

\section{B. Unequal-flavor asymmetry}

Now we consider the unequal-flavor asymmetry $A(+-,-+, \Delta t)$, which is given in general by

$$
A(+-,-+, \Delta t)=\frac{\left(|U|^{2}-|V|^{2}\right)\left(e^{-\Gamma_{L} \Delta t}-e^{-\Gamma_{S} \Delta t}\right)+4 \Im\left(U^{*} V\right) \sin (\Delta m \Delta t)}{\left(||^{2}+|V|^{2}\right)\left(e^{-\Gamma_{L} \Delta t}+e^{-\Gamma_{S} \Delta t}\right)+4 \Re\left(U^{*} V\right) \cos (\Delta m \Delta t)}
$$

with $U$ and $V$ given in (56) and (57). $A(+-,-+, \Delta t=0)$ always vanishes exactly.

If CP is conserved directly, then $R^{+}=R^{-}, S^{+}=S^{-}$, consequently

$$
\begin{aligned}
U & =\frac{1}{\sqrt{2}(1+\Omega)}\left[\left(x_{S}-x_{L}^{-1}\right) R^{+} S^{+}+\left(R^{+}\right)^{2}-\Omega\left(S^{+}\right)^{2}\right], \\
V & =\frac{1}{\sqrt{2}(1+\Omega)}\left[\left(x_{L}^{-1}-x_{S}\right) R^{+} S^{+}+\Omega\left(R^{+}\right)^{2}-\left(S^{+}\right)^{2}\right],
\end{aligned}
$$

hence $A(+-,-+, \Delta t)$ is given by (72) with the following replacement,

$$
\begin{aligned}
|U|^{2}-|V|^{2} & \rightarrow\left(1-|\Omega|^{2}\right)\left(1-\left|\zeta_{+}\right|^{4}\right)+2 \Re\left[\left(1+\Omega^{*}\right)\left(x_{S}-x_{L}^{-1}\right) \zeta_{+}\left(1-\zeta_{+}^{* 2}\right)\right]-4 \Im\left(\zeta_{+}^{* 2}\right) \Im \Omega \\
\Im\left(U^{*} V\right) & \rightarrow \Im\left[-\left(x_{S}-x_{L}^{-1}\right) \zeta_{+}\left(1+\Omega^{*}\right)\left(1-\zeta_{+}^{* 2}\right)-\zeta_{+}^{2}-|\Omega|^{2} \zeta_{+}^{* 2}+\Omega+\Omega^{*}\left|\zeta_{+}\right|^{4}\right] \\
\left.\left|U^{2}+\right| V\right|^{2} & \rightarrow 2\left|x_{S}-x_{L}^{-1}\right|^{2}\left|\zeta_{+}\right|^{2}+\left(1+|\Omega|^{2}\right)\left(1+\left|\zeta_{+}\right|^{4}\right)+2 \Re\left[\left(1-\Omega^{*}\right)\left(x_{S}-x_{L}^{-1}\right) \zeta_{+}\left(1+\zeta_{+}^{*}\right)\right]-4 \Re\left(\zeta_{+}^{2}\right) \Re \Omega \\
\Re\left(U^{*} V\right) & \rightarrow-\left|x_{S}-x_{L}^{-1}\right|^{2}\left|\zeta_{+}\right|^{2}+\Re\left[-\left(x_{S}-x_{L}^{-1}\right) \zeta_{+}\left(1-\Omega^{*}\right)\left(1+\zeta_{+}^{* 2}\right)-\zeta_{+}^{2}|\Omega|^{2} \zeta_{+}^{* 2}+\Omega_{+} \Omega^{*}\left|\zeta_{+}\right|^{4}\right] .
\end{aligned}
$$

Furthermore, if CP is conserved directly while CPT is conserved indirectly, then

$$
\begin{aligned}
U & =\frac{1}{\sqrt{2}(1+\Omega)}\left[\left(\frac{q}{p}-\frac{p}{q}\right) R^{+} S^{+}+\left(R^{+}\right)^{2}-\left(S^{+}\right)^{2}\right], \\
V & =\frac{1}{\sqrt{2}(1+\Omega)}\left[\left(\frac{p}{q}-\frac{q}{p}\right) R^{+} S^{+}+\left(R^{+}\right)^{2}-\left(S^{+}\right)^{2}\right],
\end{aligned}
$$

consequently,

$$
A(+-,-+, \Delta t)=\frac{2 \Re\left[\left(\frac{q}{p}-\frac{p}{q}\right) \zeta_{+}\left(1-\zeta_{+}^{*}\right)\right]\left(e^{-\Gamma_{L} \Delta t}-e^{-\Gamma_{S} \Delta t}\right)-4 \Im\left[\left(\frac{q}{p}-\frac{p}{q}\right) \zeta_{+}\left(1-\zeta_{+}^{* 2}\right)\right] \sin (\Delta m \Delta t)}{\left[\left|\frac{q}{p}-\frac{p}{q}\right|^{2}\left|\zeta_{+}\right|^{2}+\left|1-\zeta_{+}{ }^{2}\right|^{2}\right]\left(e^{-\Gamma_{L} \Delta t}+e^{-\Gamma_{S} \Delta t}\right)+2\left[-\left|\frac{q}{p}-\frac{p}{q}\right|^{2}\left|\zeta_{+}\right|^{2}+\left|1-\zeta_{+}\right|^{2}\right] \cos (\Delta m \Delta t)}
$$


If CPT is conserved directly, then $\left(R^{+}\right)^{*}=R^{-}$and $\left(S^{+}\right)^{*}=S^{-}$, consequently

$$
\begin{aligned}
& U=\frac{1}{\sqrt{2}(1+\Omega)}\left[-x_{L}^{-1} R^{+}\left(S^{+}\right)^{*}+x_{S}\left(R^{+}\right)^{*} S^{+}+\left|R^{+}\right|^{2}-\Omega\left|S^{+}\right|^{2}\right], \\
& V=\frac{1}{\sqrt{2}(1+\Omega)}\left[x_{L}^{-1} R^{+}\left(S^{+}\right)^{*}-x_{S}\left(R^{+}\right)^{*} S^{+}+\Omega\left|R^{+}\right|^{2}-\left|S^{+}\right|^{2}\right],
\end{aligned}
$$

hence $A(+-,-+, \Delta t)$ is given by (72) with the following replacement, $|U|^{2}-|V|^{2} \rightarrow\left(1-|\Omega|^{2}\right)\left(1-|\zeta+|^{4}\right)+2 \Re\left\{x_{S}(1+\right.$ $\left.\left.\Omega^{*}\right) \zeta_{+}-x_{L}^{-1}\left(1-\Omega^{*}\right) \zeta_{+}^{*}\right\}\left(1-|\zeta+|^{2}\right),|U|^{2}+|V|^{2} \rightarrow 2\left(\left|x_{L}^{-1}\right|^{2}+\left|x_{S}\right|^{2}-2 \Re \Omega\right)\left|\zeta_{+}\right|^{2}+\left(1+|\Omega|^{2}\right)\left(1+\left|\zeta_{+}\right|^{4}\right)-4 \Re\left(x_{L}^{-1^{*}} x_{S} \zeta_{+}^{2}\right)+$ $2 \Re\left\{\left[\left(1-\Omega^{*}\right) x_{S}-(1-\Omega) x_{L}^{-1^{*}}\right] \zeta_{+}\right\}\left(1+\left|\zeta_{+}\right|^{2}\right), U^{*} V \rightarrow-\left(1+|\Omega|^{2}+\left|x_{S}\right|^{2}+\left|x_{L}^{-1}\right|^{2}\right)\left|\zeta_{+}\right|^{2}+\Omega+\Omega^{*}\left|\zeta_{+}\right|^{4}+x_{L}^{-1^{*}} x_{S} \zeta_{+}{ }^{2}-$ $\left(\Omega x_{L}^{-1^{*}}+x_{S}\right) \zeta_{+}+\left(x_{L}^{-1^{*}}+\Omega^{*} x_{S}\right) \zeta_{+}\left|\zeta_{+}\right|^{2}+\left(\Omega x_{S}^{*}+x_{L}^{-1}\right) \zeta_{+}{ }^{*}-\left(x_{S}^{*}+\Omega^{*} x_{L}^{-1}\right) \zeta_{+}{ }^{*}\left|\zeta_{+}\right|^{2}+x_{S}^{*} x_{L}^{-1} \zeta_{+}{ }^{* 2}$.

Furthermore, if CPT is conserved directly while $\mathrm{CP}$ is conserved indirectly, then

$$
\begin{aligned}
& U=\frac{1}{2 \sqrt{2}}\left[-R^{+}\left(S^{+}\right)^{*}+S^{+}\left(R^{+}\right)^{*}+\left|R^{+}\right|^{2}-\left|S^{+}\right|^{2}\right], \\
& V=\frac{1}{\sqrt{2}}\left[R^{+}\left(S^{+}\right)^{*}-S^{+}\left(R^{+}\right)^{*}+\left|R^{+}\right|^{2}-\left|S^{+}\right|^{2}\right] .
\end{aligned}
$$

Consequently $A(+-,-+, \Delta t)$ is given by (72) under the replacement specified in the last paragraph now with $x_{L}=$ $x_{S}=\Omega=1$, therefore

$$
A(+-,-+, \Delta t)=\frac{2\left(1-\left|\zeta_{+}\right|^{2}\right) \Re\left(\zeta_{+}\right)\left(e^{-\Gamma_{L} \Delta t}-e^{-\Gamma_{S} \Delta t}\right)-4\left(1-\left|\zeta_{+}\right|^{2}\right) \Im\left(\zeta_{+}\right) \sin (\Delta m \Delta t)}{\left|1-\zeta_{+}^{2}\right|^{2}\left(e^{-\Gamma_{L} \Delta t}+e^{-\Gamma_{S} \Delta t}\right)+2\left[1+\left|\zeta_{+}\right|^{4}-4\left|\zeta_{+}\right|^{2}+2 \Re\left(\zeta_{+}^{2}\right)\right] \cos (\Delta m \Delta t)} .
$$

If both CP and CPT are conserved directly, $R^{+}=R^{-}$and $S^{+}=S^{-}$are both real numbers, consequently $U$ and $V$ are given as (73) and (74), while $A(+-,-+, \Delta t)$ is given by (72) with the replacement (75), but now with $\zeta_{+}=\zeta_{+}^{*}=\Re\left(\zeta_{+}^{*}\right)$ while $\Im\left(\zeta^{* 2}\right)=0$.

If CPT is conserved indirectly, then $x_{S}=x_{L}=q / p, \Omega=1$, hence

$$
\begin{aligned}
U & =\frac{1}{2 \sqrt{2}}\left[-\frac{p}{q} R^{+} S^{-}+\frac{q}{p} S^{+} R^{-}+R^{+} R^{-}-S^{+} S^{-}\right], \\
V & =\frac{1}{\sqrt{2}}\left[\frac{p}{q} R^{+} S^{-}-\frac{q}{p} S^{+} R^{-}+R^{+} R^{-}-S^{+} S^{-}\right] .
\end{aligned}
$$

Consequently $A(+-,-+, \Delta t)$ is given by (72) with the following replacement, $|U|^{2}-|V|^{2} \rightarrow 4 \Re\left(-\frac{p^{*}}{q^{*}} \zeta_{-}{ }^{*}+\frac{p^{*}}{q^{*}}\left|\zeta_{-}\right|^{2} \zeta_{+}+\right.$ $\left.\frac{q^{*}}{p^{*}} \zeta+^{*}-\frac{q^{*}}{p^{*}}\left|\zeta_{+}\right|^{2} \zeta_{-}\right),|U|^{2}+|V|^{2} \rightarrow 2\left(\left|\frac{p}{q} \zeta_{-}\right|^{2}+\left|\frac{q}{p} \zeta_{+}\right|^{2}+1+\left|\zeta_{+} \zeta_{-}\right|^{2}\right)-4 \Re\left(\frac{p^{*} q}{q^{*} p} \zeta_{-}{ }^{*} \zeta_{+}+\zeta_{+} \zeta_{-}\right), \Re\left(U^{*} V\right) \rightarrow 1-\left|\frac{p}{q} \zeta_{-}\right|^{2}-$ $\left|\frac{q}{p} \zeta_{+}\right|^{2}+\left|\zeta_{+} \zeta_{-}\right|^{2}+2 \Re\left(\frac{p^{*} q}{q^{*} p} \zeta_{+} \zeta_{-}{ }^{*}-\zeta_{+} \zeta_{-}\right), \Im\left(U^{*} V\right) \rightarrow 2 \Im\left(\frac{p}{q} \zeta_{-}+\frac{p^{*}}{q^{*}}\left|\zeta_{-}\right|^{2} \zeta_{+}-\frac{q}{p} \zeta_{+}-\frac{q^{*}}{p^{*}}\left|\zeta_{+}\right|^{2} \zeta_{-}\right)$.

If CP is conserved indirectly, then no matter whether CPT is conserved indirectly, $x_{S}=x_{L}=\Omega=1$, consequently $U$ and $V$ are given by (84) and (85) with $p / q=1$, hence $A(+-,-+, \Delta t)$ is given by (72) with the following replacement, $|U|^{2}-|V|^{2} \rightarrow 4 \Re\left(-\zeta_{-}{ }^{*}+\left|\zeta_{-}\right|^{2} \zeta_{+}+\zeta_{+}{ }^{*}-\left|\zeta_{+}\right|^{2} \zeta_{-}\right),|U|^{2}+|V|^{2} \rightarrow 2\left(\left|\zeta_{-}\right|^{2}+\left|\zeta_{+}\right|^{2}+1+\left|\zeta_{+} \zeta_{-}\right|^{2}\right)-8 \Re\left(\zeta_{-}\right) \Re\left(\zeta_{+}\right)$, $\Re\left(U^{*} V\right) \rightarrow 1-\left|\zeta_{-}\right|^{2}-\left|\zeta_{+}\right|^{2}+\left|\zeta_{+} \zeta_{-}\right|^{2}+4 \Im\left(\zeta_{+}\right) \Im\left(\zeta_{-}\right), \Im\left(U^{*} V\right) \rightarrow 2 \Im\left(\zeta_{-}+\left|\zeta_{-}\right|^{2} \zeta_{+}-\zeta_{+}-\left|\zeta_{+}\right|^{2} \zeta_{-}\right)$.

If $\mathrm{CP}$ is conserved both directly and indirectly,

$$
U=V=\frac{1}{2 \sqrt{2}}\left[\left(R^{+}\right)^{2}-\left(S^{+}\right)^{2}\right]
$$

which, according to (72), implies

$$
A(+-,-+, \Delta t)=0
$$

Hence we have the following exact theorem.

Theorem 3 If the unequal-flavor asymmetry $A(+-,-+, \Delta t) \neq 0$, then CP must be violated, directly or indirectly or both.

If CPT is conserved both directly and indirectly, then

$$
\begin{aligned}
U & =\frac{1}{2 \sqrt{2}}\left[-\frac{p}{q} R^{+}\left(S^{+}\right)^{*}+\frac{q}{p}\left(R^{+}\right)^{*} S^{+}+\left|R^{+}\right|^{2}-\left|S^{+}\right|^{2}\right], \\
V & =\frac{1}{2 \sqrt{2}}\left[\frac{p}{q} R^{+}\left(S^{+}\right)^{*}-\frac{q}{p}\left(R^{+}\right)^{*} S^{+}+\left|R^{+}\right|^{2}-\left|S^{+}\right|^{2}\right] .
\end{aligned}
$$


Consequently, in obtaining $A(+-,-+, \Delta t)$, the replacement for the four functions of $U$ and $V$ is given by that for the case of direct CPT conservation with $\zeta_{-}=\zeta_{+}^{*}$. Therefore

$$
A(+-,-+, \Delta t)=\frac{\left(1-\left|\zeta_{+}\right|^{2}\right)\left\{-2 \Re\left[\left(\frac{p}{q}-\frac{q^{*}}{p^{*}}\right) \zeta_{-}\right]\left(e^{-\Gamma_{L} \Delta t}-e^{-\Gamma_{S} \Delta t}\right)+4 \Im\left[\left(\frac{p}{q}+\frac{q^{*}}{p^{*}}\right) \zeta_{-}\right] \sin (\Delta m \Delta t)\right.}{E_{+}\left(e^{-\Gamma_{L} \Delta t}+e^{-\Gamma_{S} \Delta t}\right)+2 E_{-} \cos (\Delta m \Delta t)},
$$

where $E_{ \pm}=\left(1-\left|\zeta_{+}\right|^{2}\right)^{2} \pm\left|\frac{p}{q} \zeta_{-}-\frac{q}{p} \zeta_{+}\right|^{2}$. Therefore we have the following exact theorem.

Theorem 4 If the unequal-flavor asymmetry $A(+-,-+, \Delta t) \neq 0$ for $\Delta t \neq 0$ while CPT is assumed to be conserved both directly and indirectly, then we can draw the following conclusions: (1) $\left|\zeta_{+}\right|=\left|\zeta_{-}\right| \neq 1$, i.e., $\left|\left\langle l^{+}|\mathcal{H}| \bar{M}_{0}\right\rangle\right|=$ $\left|\left\langle l^{-}|\mathcal{H}| M^{0}\right\rangle\right| \neq\left|\left\langle l^{+}|\mathcal{H}| M^{0}\right\rangle\right|=\left|\left\langle l^{-}|\mathcal{H}| \bar{M}_{0}\right\rangle\right|$; (2) moreover, $\zeta_{-}=\zeta_{+}^{*} \neq 0$, i.e., $\left\langle l^{-}|\mathcal{H}| M^{0}\right\rangle=\left\langle l^{+}|\mathcal{H}| \bar{M}^{0}\right\rangle^{*} \neq 0$, which means $\Delta \mathcal{F}=\Delta Q$ rule must be violated.

\section{DECAYS INTO CP EIGENSTATES}

Now we consider the situation that the decay products of Alice and Bob are both CP eigenstates, as in KLOE experiment, where the rate of both kaons decaying to $\pi^{+} \pi^{-}$was obtained up to a proportional factor [8, 9]. We generically denote the CP eigenstates as $\left|h^{+}\right\rangle$with eigenvalue +1 and $\left|h^{-}\right\rangle$with eigenvalue -1 . Examples for $\left|h^{+}\right\rangle$ include $\pi^{+} \pi^{-}, \pi^{0} \pi^{0}$, etc. Examples for $\left|h^{-}\right\rangle$include $\pi^{0} \pi^{0} \pi^{0}$, etc. For convenience of discussions, we introduce the parameters defined as

$$
w_{ \pm \alpha}^{x} \equiv\left\langle h_{\alpha}^{x}\left|\mathcal{H}_{\alpha}\right| M_{ \pm}\right\rangle_{\alpha},
$$

where $x= \pm 1$. These parameters were not introduced previously, because an isolated single meson cannot be in the state $\left|M_{ \pm}\right\rangle$physically. However, the entangled state $\left|\Psi_{-}\right\rangle$renders the introduction of these parameters meaningful and convenient, because the entangled state can be exactly written in terms of the CP basis, as in (34), hence an entangled pair of mesons decay from superposition of direct products of $\mathrm{CP}$ eigenstates. No that there is no tagging in CP basis, only the final decay products, e.g. pions, are measured. Therefore the use of parameter $w_{ \pm \alpha}^{x}$ is legitimate. This is a remarkable point we would like to exploit. The parameter $w_{ \pm}^{x}$ may be measured by using entangled mesons. They cannot be directly measured by using single mesons, as there is no way to prepare a single meson in a $\mathrm{CP}$ eigenstate. Nevertheless, by using (1), we have

$$
w_{ \pm}^{x} \equiv \frac{1}{\sqrt{2}}\left(\left\langle h^{x}|\mathcal{H}| M^{0}\right\rangle \pm\left\langle h^{x}|\mathcal{H}| \bar{M}^{0}\right\rangle\right),
$$

where each term on the RHS can be measured for isolated single mesons. Analogously, $w_{ \pm}^{x}$ can also be related to parameters for mass basis, by using (12),

$$
\begin{aligned}
w_{+}^{x} & =\frac{1}{1-\epsilon_{S} \epsilon_{L}}\left(\sqrt{1+\left|\epsilon_{S}\right|^{2}}\left\langle h^{x}|\mathcal{H}| M_{S}\right\rangle-\epsilon_{S} \sqrt{1+\left|\epsilon_{L}\right|^{2}}\left\langle h^{x}|\mathcal{H}| M_{L}\right\rangle\right), \\
w_{-}^{x} & =\frac{1}{1-\epsilon_{S} \epsilon_{L}}\left(\sqrt{1+\left|\epsilon_{L}\right|^{2}}\left\langle h^{x}|\mathcal{H}| M_{L}\right\rangle-\epsilon_{L} \sqrt{1+\left|\epsilon_{S}\right|^{2}}\left\langle h^{x}|\mathcal{H}| M_{S}\right\rangle\right),
\end{aligned}
$$

where each term on the RHS of each identity can be measured for isolated single mesons.

One can calculate the amplitude of such a joint decay in which Alice decays to $\left|h_{a}^{x}\right\rangle$ at $t_{a}$ while Bob decays to $\left|h_{b}^{y}\right\rangle$ at $t_{b}$, where $x$ and $y$ each represents \pm 1 ,

$$
\begin{aligned}
\left\langle h_{a}^{x}, h_{b}^{y}\left|\mathcal{H}_{a} \mathcal{H}_{b}\right| \Psi_{-}\left(t_{a}, t_{b}\right)\right\rangle & =\frac{1}{\sqrt{2}}\left(\left\langle h_{a}^{x}\left|\mathcal{H}_{a}\right| M^{+}\left(t_{a}\right)\right\rangle_{a}\left\langle h_{b}^{y}\left|\mathcal{H}_{b}\right| M_{-}\left(t_{b}\right)\right\rangle_{b}-\left\langle h_{a}^{x}\left|\mathcal{H}_{a}\right| M^{-}\left(t_{a}\right)\right\rangle_{a}\left\langle h_{b}^{y}\left|\mathcal{H}_{b}\right| M_{+}\left(t_{b}\right)\right\rangle_{b}\right) \\
& =M\left(h_{a}^{x}, h_{b}^{y}\right) e^{-i\left(\lambda_{S} t_{a}+\lambda_{L} t_{b}\right)}+N\left(h_{a}^{x}, h_{b}^{y}\right) e^{-i\left(\lambda_{L} t_{a}+\lambda_{S} t_{b}\right)}
\end{aligned}
$$

where $M\left(h_{a}^{x}, h_{b}^{y}\right) \equiv\left[-\left(1-x_{L}^{-1}+x_{S}-\Omega\right) w_{+a}^{x} w_{+b}^{y}+\left(1+x_{L}^{-1}+x_{S}+\Omega\right) w_{+a}^{x} w_{-b}^{y}-\left(1-x_{L}^{-1}-x_{S}+\Omega\right) w_{-a}^{x} w_{+b}^{y}+(1+\right.$ $\left.\left.x_{L}^{-1}-x_{S}-\Omega\right) w_{-a}^{x} w_{-b}^{y}\right] /[2 \sqrt{2}(1+\Omega)], N\left(h_{a}^{x}, h_{b}^{y}\right) \equiv\left[\left(1-x_{L}^{-1}+x_{S}-\Omega\right) w_{+a}^{x} w_{+b}^{y}+\left(1-x_{L}^{-1}-x_{S}+\Omega\right) w_{+a}^{x} w_{-b}^{y}-(1+\right.$ $\left.\left.x_{L}^{-1}+x_{S}+\Omega\right) w_{-a}^{x} w_{+b}^{y}-\left(1+x_{L}^{-1}-x_{S}-\Omega\right) w_{-a}^{x} w_{-b}^{y}\right] /[2 \sqrt{2}(1+\Omega)]$.

Therefore we obtain the joint rate

$$
\begin{aligned}
I\left(h_{a}^{x}, t_{a} ; h_{b}^{y}, t_{b}\right)= & e^{-\left(\Gamma_{S}+\Gamma_{L}\right) t_{a}}\left\{|M|^{2} e^{-\Gamma_{L} \Delta t}+|N|^{2} e^{-\Gamma_{S} \Delta t}\right. \\
& \left.+2 e^{-\frac{\Gamma_{S}+\Gamma_{L}}{2} \Delta t}\left[\Re\left(M^{*} N\right) \cos (\Delta m \Delta t)+\Im\left(M^{*} N\right) \sin (\Delta m \Delta t)\right]\right\},
\end{aligned}
$$


where $M \equiv M\left(h_{a}^{x}, h_{b}^{y}\right), N \equiv N\left(h_{a}^{x}, h_{b}^{y}\right)$.

In experiments, it is more convenient to use the integrated rate

$$
I^{\prime}\left(h_{a}^{x}, h_{b}^{y}, \Delta t\right)=\int_{0}^{\infty} I\left(h_{a}^{x}, t_{a} ; h_{b}^{y}, t_{a}+\Delta t\right) d t_{a},
$$

which is simply given by $I\left(l_{a}^{x}, t_{a} ; l_{b}^{y}, t_{a}+\Delta t\right)$ as in (58) with $e^{-\left(\Gamma_{S}+\Gamma_{L}\right) t_{a}}$ replaced as $1 /\left(\Gamma_{S}+\Gamma_{L}\right)$.

We focus on the situation that $\left|h_{a}^{+}\right\rangle=\left|h_{b}^{+}\right\rangle=\left|h^{+}\right\rangle$and $\left|h_{a}^{-}\right\rangle=\left|h_{b}^{-}\right\rangle=\left|h^{-}\right\rangle$, hence

$$
\begin{aligned}
& w_{+a}^{+}=w_{+b}^{+} \equiv Q^{+}, \\
& w_{-a}^{+}=w_{-b}^{+} \equiv X^{+}, \\
& w_{+a}^{-}=w_{+b}^{-} \equiv X^{-}, \\
& w_{-a}^{-}=w_{-b}^{-} \equiv Q^{-} .
\end{aligned}
$$

It is straightforward to find the following properties. If CP is conserved directly, then $X^{ \pm}=0$, hence $X^{ \pm}$is a parameter characterizing the direct $\mathrm{CP}$ violation. If CPT is conserved directly, then $X^{ \pm}$is purely imaginary, i.e. $X^{ \pm}=-X^{ \pm *}$.

We define

$$
\xi_{ \pm} \equiv \frac{X^{ \pm}}{Q^{ \pm}}
$$

$Q^{ \pm}$and $X^{ \pm}$are not directly measurable quantities, as $M_{S}$ and $M_{L}$, rather than $M_{+}$and $M_{-}$, are physical. However, from (77) and (8), we have

$$
\begin{aligned}
Q^{+}+\epsilon_{S} X^{+} & =\sqrt{1+\left|\epsilon_{S}\right|^{2}}\left\langle h^{+}|\mathcal{H}| M_{S}\right\rangle, \\
\epsilon_{L} X^{-}+Q^{-} & =\sqrt{1+\left|\epsilon_{L}\right|^{2}}\left\langle h^{-}|\mathcal{H}| M_{L}\right\rangle .
\end{aligned}
$$

Therefore,

$$
\begin{aligned}
\eta_{h^{+}} & \equiv \frac{\left\langle h^{+}|\mathcal{H}| M_{L}\right\rangle}{\left\langle h^{+}|\mathcal{H}| M_{S}\right\rangle}=\frac{\xi^{+}+\epsilon_{L}}{1+\epsilon_{S} \xi^{+}}, \\
\eta_{h^{-}} & \equiv \frac{\left\langle h^{-}|\mathcal{H}| M_{S}\right\rangle}{\left\langle h^{-}|\mathcal{H}| M_{L}\right\rangle}=\frac{\xi^{-}+\epsilon_{S}}{1+\epsilon_{L} \xi^{-}} .
\end{aligned}
$$

In the case of $\left|h^{+}\right\rangle=\left|\pi^{+} \pi^{-}\right\rangle,\left|\eta_{h^{+}}\right\rangle$is just the well-known

$$
\eta_{+-}=\frac{\left\langle\pi^{+} \pi^{-}|\mathcal{H}| M_{L}\right\rangle}{\left\langle\pi^{+} \pi^{-}|\mathcal{H}| M_{S}\right\rangle} .
$$

In the case of $\left|h^{+}\right\rangle=\left|\pi^{0} \pi^{0}\right\rangle,\left|\eta_{h^{+}}\right\rangle$is just the well-known

$$
\eta_{00}=\frac{\left\langle\pi^{0} \pi^{0}|\mathcal{H}| M_{L}\right\rangle}{\left\langle\pi^{0} \pi^{0}|\mathcal{H}| M_{S}\right\rangle}
$$

One obtains

$$
\begin{aligned}
M\left(h^{+}, h^{+}\right)= & -N\left(h^{+}, h^{+}\right)=\frac{1}{2 \sqrt{2}(1+\Omega)}\left[-\left(1-x_{L}^{-1}+x_{S}-\Omega\right)\left(Q^{+}\right)^{2}+2\left(x_{L}^{-1}+x_{S}\right) Q^{+} X^{+}\right. \\
& \left.+\left(1+x_{L}^{-1}-x_{S}-\Omega\right)\left(X^{+}\right)^{2}\right], \\
M\left(h^{-}, h^{-}\right)= & -N\left(h^{-}, h^{-}\right)=\frac{1}{2 \sqrt{2}(1+\Omega)}\left[-\left(1-x_{L}^{-1}+x_{S}-\Omega\right)\left(X^{-}\right)^{2}+2\left(x_{L}^{-1}+x_{S}\right) X^{-} Q^{-}\right. \\
& \left.+\left(1+x_{L}^{-1}-x_{S}-\Omega\right)\left(Q^{-}\right)^{2}\right], \\
M\left(h^{+}, h^{-}\right)= & -M\left(h^{-}, h^{+}\right) \\
= & \frac{1}{2 \sqrt{2}(1+\Omega)}\left[\left(1+x_{S}\right) Q^{+}+\left(1-x_{S}\right) X^{+}\right]\left[\left(1+x_{L}^{-1}\right) Q^{-}-\left(1-x_{L}^{-1}\right) X^{-}\right] \equiv Z, \\
N\left(h^{+}, h^{-}\right)= & -N\left(h^{-}, h^{+}\right) \\
= & \frac{1}{2 \sqrt{2}(1+\Omega)}\left[\left(1-x_{L}^{-1}\right) Q^{+}-\left(1+x_{L}^{-1}\right) X^{+}\right]\left[\left(1-x_{S}\right) Q^{-}+\left(1+x_{S}\right) X^{-}\right] \equiv Y .
\end{aligned}
$$


Therefore, we obtain

$$
\begin{aligned}
& \begin{aligned}
I\left(h^{+}, t_{a} ; h^{+}, t_{b}\right)= & \frac{\left|-\left(1-x_{L}^{-1}+x_{S}-\Omega\right)\left(Q^{+}\right)^{2}+2\left(x_{L}^{-1}+x_{S}\right) Q^{+} X^{+}+\left(1+x_{L}^{-1}-x_{S}-\Omega\right)\left(X^{+}\right)^{2}\right|^{2}}{8|1+\Omega|^{2}} \\
& \times e^{-\left(\Gamma_{S}+\Gamma_{L}\right) t_{a}}\left[e^{-\Gamma_{S} \Delta t}+e^{-\Gamma_{L} \Delta t}-2 e^{-\frac{1}{2}\left(\Gamma_{S}+\Gamma_{L}\right) \Delta t} \cos (\Delta m \Delta t)\right],
\end{aligned} \\
& \begin{aligned}
I\left(h^{-}, t_{a} ; h^{-}, t_{b}\right)= & \frac{\left|-\left(1-x_{L}^{-1}+x_{S}-\Omega\right)\left(X^{-}\right)^{2}+2\left(x_{L}^{-1}+x_{S}\right) X^{-} Q^{-}+\left(1+x_{L}^{-1}-x_{S}-\Omega\right)\left(Q^{-}\right)^{2}\right|^{2}}{8|1+\Omega|^{2}} \\
& \times e^{-\left(\Gamma_{S}+\Gamma_{L}\right) t_{a}}\left[e^{-\Gamma_{S} \Delta t}+e^{-\Gamma_{L} \Delta t}-2 e^{-\frac{1}{2}\left(\Gamma_{S}+\Gamma_{L}\right) \Delta t} \cos (\Delta m \Delta t)\right],
\end{aligned} \\
& I\left(h^{+}, t_{a} ; h^{-}, t_{b}\right)=e^{-\left(\Gamma_{S}+\Gamma_{L}\right) t_{a}}\left\{|Z|^{2} e^{-\Gamma_{L} \Delta t}+|Y|^{2} e^{-\Gamma_{S} \Delta t}+2 e^{-\frac{\Gamma_{S}+\Gamma_{L}}{2} \Delta t}\left[\Re\left(Z^{*} Y\right) \cos (\Delta m \Delta t)+\Im\left(Z^{*} Y\right) \sin (\Delta m \Delta t)\right]\right\}, \\
& I\left(h^{-}, t_{a} ; h^{+}, t_{b}\right)=e^{-\left(\Gamma_{S}+\Gamma_{L}\right) t_{a}}\left\{|Y|^{2} e^{-\Gamma_{L} \Delta t}+|Z|^{2} e^{-\Gamma_{S} \Delta t}+2 e^{-\frac{\Gamma_{S}+\Gamma_{L}}{2} \Delta t}\left[\Re\left(Z^{*} Y\right) \cos (\Delta m \Delta t)-\Im\left(Z^{*} Y\right) \sin (\Delta m \Delta t)\right]\right\} \text {. }
\end{aligned}
$$

One can also consider the integrated rate

$$
I^{\prime}\left(h^{x}, h^{y}, \Delta t\right)=\int_{0}^{\infty} I\left(h^{x}, t_{a} ; h^{y}, t_{a}+\Delta t\right) d t_{a}
$$

which is simply given by $I\left(h^{x}, t_{a} ; h^{y}, t_{a}+\Delta t\right)$ as in (112), with $e^{-\left(\Gamma_{S}+\Gamma_{L}\right) t_{a}}$ replaced as $1 /\left(\Gamma_{S}+\Gamma_{L}\right)$.

\section{CP AND CPT VIOLATIONS IN DECAYS IN CP BASIS}

First, it can be seen that the equal-CP decay rates $I\left[h^{+}, t_{a} ; h^{+}, t_{b}\right]$ and $I\left[h^{-}, t_{a} ; h^{-}, t_{b}\right]$ are both proportional $e^{-\Gamma_{S} \Delta t}+e^{-\Gamma_{L} \Delta t}-2 e^{-\frac{1}{2}\left(\Gamma_{S}+\Gamma_{L}\right) \Delta t} \cos (\Delta m \Delta t)$, as demonstrated by KLOE experimental data 8, 9].

For $\Delta t=0$, we always have $I\left[h^{+}, t_{a} ; h^{+}, t_{a}\right]=\left[h^{-}, t_{a} ; h^{-}, t_{a}\right]=I^{\prime}\left[h^{+}, h^{+}, 0\right]=\left[h^{-}, h^{-}, 0\right]=0$ no matter whether $\mathrm{CP}$ or $\mathrm{CPT}$ is violated.

The four joint rates in (112) or (113) can form some asymmetries between different modes of decays into CP eigenstates. As $I^{\prime}\left(h^{x}, h^{y}, \Delta t\right) e^{-\left(\Gamma_{L}+\Gamma_{S}\right) t_{a}}=\frac{1}{\Gamma_{L}+\Gamma_{S}} I\left(h^{x}, t_{a} ; h^{y}, t_{b}\right)$, the asymmetries defined for the instantaneous joint probability $I$ and its integration $I^{\prime}$ for a specific $\Delta t$ are equal, and depend only on $\Delta t \equiv t_{b}-t_{a}$,

$$
B\left(h^{x} h^{y}, h^{y} h^{x}, \Delta t\right) \equiv \frac{I\left[h^{x}, t_{a} ; h^{y}, t_{a}+\Delta t\right]-I\left[h^{y}, t_{a} ; h^{x}, t_{a}+\Delta t\right]}{I\left[h^{x}, t_{a} ; h^{y}, t_{a}+\Delta t\right]+I\left[h^{y}, t_{a} ; h^{x}, t_{a}+\Delta t\right]}=\frac{I^{\prime}\left[h^{x}, h^{y}, \Delta t\right]-I^{\prime}\left[h^{y}, h^{x}, \Delta t\right]}{I^{\prime}\left[h^{x}, h^{y}, \Delta t\right]+I^{\prime}\left[h^{y}, h^{x}, \Delta t\right]},
$$

which is always time-independent.

In general, the equal-CP asymmetry is found to be

$$
B(++,--, \Delta t)=\frac{P\left(Q^{+}, X^{+}\right)-P\left(X^{-}, Q^{-}\right)}{P\left(Q^{+}, X^{+}\right)+P\left(X^{-}, Q^{-}\right)}
$$

where

$$
P(\beta, \gamma) \equiv\left|-\left(1-x_{L}^{-1}+x_{S}-\Omega\right) \beta^{2}+2\left(x_{L}^{-1}+x_{S}\right) \beta \gamma+\left(1+x_{L}^{-1}-x_{S}-\Omega\right) \gamma^{2}\right|^{2} .
$$

Theorem 5 The equal-CP asymmetry $B(++,--, \Delta t)$ is always a constant independent of $\Delta t$.

In general, the unequal-CP asymmetry is found to be

$$
\begin{aligned}
B(+-,-+, \Delta t) & =\frac{\left(|Z|^{2}-|Y|^{2}\right)\left(e^{-\Gamma_{L} \Delta t}-e^{-\Gamma_{S} \Delta t}\right)+4 e^{-\frac{\Gamma_{S}+\Gamma_{L}}{2} \Delta t} \Im\left(Z^{*} Y\right) \sin (\Delta m \Delta t)}{\left(|Z|^{2}+|Y|^{2}\right)\left(e^{-\Gamma_{L} \Delta t}+e^{-\Gamma_{S} \Delta t}\right)+4 e^{-\frac{\Gamma_{S}+\Gamma_{L}}{2} \Delta t} \Re\left(Z^{*} Y\right) \cos (\Delta m \Delta t)} \\
& =\frac{\left(|\tilde{Z}|^{2}-|\tilde{Y}|^{2}\right)\left(e^{-\Gamma_{L} \Delta t}-e^{-\Gamma_{S} \Delta t}\right)+4 e^{-\frac{\Gamma_{S}+\Gamma_{L}}{2} \Delta t} \Im(\tilde{Z} * \tilde{Y}) \sin (\Delta m \Delta t)}{\left(|\tilde{Z}|^{2}+|\tilde{Y}|^{2}\right)\left(e^{-\Gamma_{L} \Delta t}+e^{-\Gamma_{S} \Delta t}\right)+4 e^{-\frac{\Gamma_{S}+\Gamma_{L}}{2} \Delta t} \Re(\tilde{Z} * \tilde{Y}) \cos (\Delta m \Delta t)},
\end{aligned}
$$

where

$$
\begin{aligned}
\tilde{Z} & \equiv\left[\left(1+x_{S}\right)+\left(1-x_{S}\right) \xi^{+}\right]\left[\left(1+x_{L}^{-1}\right)-\left(1-x_{L}^{-1}\right) \xi^{-}\right] \\
\tilde{Y} & \equiv\left[\left(1-x_{L}^{-1}\right)-\left(1+x_{L}^{-1}\right) \xi^{+}\right]\left[\left(1-x_{S}\right)+\left(1+x_{S}\right) \xi^{-}\right]
\end{aligned}
$$

When $\Delta t=0$, the unequal-CP asymmetry is always 0 no matter whether $\mathrm{CP}$ or CPT is violated. Therefore we have the following result.

Theorem 6 For $\Delta t=0$, the unequal-CP asymmetry $B(+-,-+, 0)$ vanishes, no matter whether $C P$ or $C P T$ is violated. 
If $\mathrm{CP}$ is conserved directly, then $X^{ \pm}=0$, consequently $B(++,--, \Delta t)$ reduces to

$$
B(++,--, \Delta t)=\frac{\left|Q^{+}\right|^{4}\left|\left(1-x_{L}^{-1}\right)\left(1+x_{S}\right)\right|^{2}-\left|Q^{-}\right|^{4}\left|\left(1+x_{L}^{-1}\right)\left(1-x_{S}\right)\right|^{2}}{\left|Q^{+}\right|^{4}\left|\left(1-x_{L}^{-1}\right)\left(1+x_{S}\right)\right|^{2}+\left|Q^{-}\right|^{4}\left|\left(1+x_{L}^{-1}\right)\left(1-x_{S}\right)\right|^{2}} .
$$

while $B(+-,-+, \Delta t)$ reduces to

$$
B(+-,-+, \Delta t)=\frac{\left(1-|W|^{2}\right)\left(e^{-\Gamma_{L} \Delta t}-e^{-\Gamma_{S} \Delta t}\right)+4 e^{-\frac{\Gamma_{S}+\Gamma_{L}}{2} \Delta t} \Im(W) \sin (\Delta m \Delta t)}{\left(1+|W|^{2}\right)\left(e^{-\Gamma_{L} \Delta t}+e^{-\Gamma_{S} \Delta t}\right)+4 e^{-\frac{\Gamma_{S}+\Gamma_{L}}{2} \Delta t} \Re(W) \cos (\Delta m \Delta t)},
$$

where $W \equiv \frac{\left(1-x_{L}^{-1}\right)\left(1-x_{S}\right)}{\left(1+x_{L}^{-1}\right)\left(1+x_{S}\right)}$.

If CP is conserved indirectly, no matter whether CPT is conserved indirectly, we have $x_{S}=x_{L}^{-1}=1$. Consequently, $M\left(h^{+}, h^{+}\right)=-N\left(h^{+}, h^{+}\right)=\frac{1}{\sqrt{2}} Q^{+} X^{+}, M\left(h^{-}, h^{-}\right)=-N\left(h^{-}, h^{-}\right)=\frac{1}{\sqrt{2}} X^{-} Q^{-}, Z=M\left(h^{+}, h^{-}\right)=-M^{-}\left(h^{-}, h^{+}\right)=$ $\frac{1}{\sqrt{2}} Q^{+} Q^{-}, Y=N\left(h^{+}, h^{-}\right)=-N\left(h^{-}, h^{+}\right)=-\frac{1}{\sqrt{2}} X^{+} X^{-}$. Therefore

$$
\begin{aligned}
I\left(h^{+}, t_{a} ; h^{+}, t_{b}\right)= & \frac{\left|Q^{+} X^{+}\right|^{2}}{2} e^{-\left(\Gamma_{S}+\Gamma_{L}\right) t_{a}}\left[e^{-\Gamma_{S} \Delta t}+e^{-\Gamma_{L} \Delta t}-2 e^{-\frac{1}{2}\left(\Gamma_{S}+\Gamma_{L}\right) \Delta t} \cos (\Delta m \Delta t)\right] \\
I\left(h^{-}, t_{a} ; h^{-}, t_{b}\right)= & \frac{\left|Q^{-} X^{-}\right|^{2}}{2} e^{-\left(\Gamma_{S}+\Gamma_{L}\right) t_{a}}\left[e^{-\Gamma_{S} \Delta t}+e^{-\Gamma_{L} \Delta t}-2 e^{-\frac{1}{2}\left(\Gamma_{S}+\Gamma_{L}\right) \Delta t} \cos (\Delta m \Delta t)\right], \\
I\left(h^{+}, t_{a} ; h^{-}, t_{b}\right)= & \frac{1}{2} e^{-\left(\Gamma_{S}+\Gamma_{L}\right) t_{a}}\left\{\left|Q^{+} Q^{-}\right|^{2} e^{-\Gamma_{L} \Delta t}+\left|X^{+} X^{-}\right|^{2} e^{-\Gamma_{S} \Delta t}\right. \\
& \left.+2 e^{-\frac{\Gamma_{S}+\Gamma_{L}}{2} \Delta t}\left[\Re\left(\left(Q^{+} Q^{-}\right)^{*} X^{+} X^{-}\right) \cos (\Delta m \Delta t)+\Im\left(\left(Q^{+} Q^{-}\right)^{*} X^{+} X^{-}\right) \sin (\Delta m \Delta t)\right]\right\} \\
I\left(h^{-}, t_{a} ; h^{+}, t_{b}\right)= & \frac{1}{2} e^{-\left(\Gamma_{S}+\Gamma_{L}\right) t_{a}}\left\{\left|X^{+} X^{-}\right|^{2} e^{-\Gamma_{L} \Delta t}+\left|Q^{+} Q^{-}\right|^{2} e^{-\Gamma_{S} \Delta t}\right. \\
& \left.+2 e^{-\frac{\Gamma_{S}+\Gamma_{L}}{2} \Delta t}\left[\Re\left(\left(Q^{+} Q^{-}\right)^{*} X^{+} X^{-}\right) \cos (\Delta m \Delta t)-\Im\left(\left(Q^{+} Q^{-}\right)^{*} X^{+} X^{-}\right) \sin (\Delta m \Delta t)\right]\right\},
\end{aligned}
$$

Therefore if CP is conserved indirectly, no matter whether CPT is conserved indirectly, the equal-CP asymmetry is simplified to

$$
B(++,--, \Delta t)=\frac{\left|Q^{+} X^{+}\right|^{2}-\left|Q^{-} X^{-}\right|^{2}}{\left|Q^{+} X^{+}\right|^{2}+\left|Q^{-} X^{-}\right|^{2}}
$$

while the unequal-CP asymmetry is simplified to

$$
B(+-,-+, \Delta t)=\frac{\left(1-\left|\xi^{+} \xi^{-}\right|^{2}\right)\left(e^{-\Gamma_{L} \Delta t}-e^{-\Gamma_{S} \Delta t}\right)+4 e^{-\frac{\Gamma_{S}+\Gamma_{L}}{2} \Delta t} \Im\left(\xi^{+} \xi^{-}\right) \sin (\Delta m \Delta t)}{\left(1+\left|\xi^{+} \xi^{-}\right|^{2}\right)\left(e^{-\Gamma_{L} \Delta t}+e^{-\Gamma_{S} \Delta t}\right)+4 e^{-\frac{\Gamma_{S}+\Gamma_{L}}{2} \Delta t} \Re\left(\xi^{+} \xi^{-}\right) \cos (\Delta m \Delta t)} .
$$

If $\mathrm{CP}$ is conserved both directly and indirectly, no matter whether CPT is conserved or not, we have $X^{ \pm}=0$, $x_{S}=x_{L}^{-1}=1$, then

$$
\begin{aligned}
& I\left(h_{a}^{+}, t_{a} ; h_{b}^{+}, t_{b}\right)=0 \\
& I\left(h_{a}^{-}, t_{a} ; h_{b}^{-}, t_{b}\right)=0 \\
& I\left(h_{a}^{+}, t_{a} ; h_{b}^{-}, t_{b}\right)=\frac{\left|Q^{+} Q^{-}\right|^{2}}{2} e^{-\left(\Gamma_{S}+\Gamma_{L}\right) t_{a}} e^{-\Gamma_{L} \Delta t} \\
& I\left(h^{-}, t_{a} ; h^{+}, t_{b}\right)=\frac{\left|Q^{+} Q^{-}\right|^{2}}{2} e^{-\left(\Gamma_{S}+\Gamma_{L}\right) t_{a}} e^{-\Gamma_{S} \Delta t}
\end{aligned}
$$

As $I\left(h_{a}^{+}, t_{a} ; h_{b}^{+}, t_{b}\right)=I\left(h_{a}^{-}, t_{a} ; h_{b}^{-}, t_{b}\right)=I^{\prime}\left(h_{a}^{+}, h_{b}^{+}, \Delta t\right)=I\left(h_{a}^{-}, h_{b}^{-}, \Delta t\right)=0$, it is meaningless to define equal-CP asymmetry in this case. In this case, the unequal-CP asymmetry is

$$
B(+-,-+, \Delta t)=\frac{e^{-\Gamma_{L} \Delta t}-e^{-\Gamma_{S} \Delta t}}{e^{-\Gamma_{L} \Delta t}+e^{-\Gamma_{S} \Delta t}},
$$

Theorem 7 If any equal-CP joint decay rate is nonzero, then CP must be violated, directly or indirectly or both.

If CPT is conserved indirectly, then $\delta_{M}=0$, thus $x_{S}=x_{L}=q / p$, and thus $\Omega=1$, consequently the equal-CP asymmetry is given by (115) with $P(\beta, \gamma)$ simplified to

$$
P(\beta, \gamma) \equiv\left|2\left(\frac{p}{q}+\frac{q}{p}\right) \beta \gamma+\left(\frac{p}{q}-\frac{q}{p}\right)\left(\gamma^{2}-\beta^{2}\right)\right|^{2} .
$$

The unequal-CP asymmetry $B(+-,-+, \Delta t)$ is given by (118) with $\tilde{Z}$ and $\tilde{Y}$ reduced to

$$
\begin{aligned}
\tilde{Z} & \equiv\left[\left(1+\frac{q}{p}\right)+\left(1-\frac{q}{p}\right) \xi^{+}\right]\left[\left(1+\frac{p}{q}\right)-\left(1-\frac{p}{q}\right) \xi^{-}\right] \\
\tilde{Y} & \equiv\left[\left(1-\frac{p}{q}\right)-\left(1+\frac{p}{q}\right) \xi^{+}\right]\left[\left(1-\frac{q}{p}\right)+\left(1+\frac{q}{p}\right) \xi^{-}\right] .
\end{aligned}
$$


If CPT is conserved indirectly while CP is conserved directly, $x_{S}=x_{L}=q / p$ while $X^{ \pm}=0$, then the equal-CP asymmetry reduces to

$$
B(++,--, \Delta t)=\frac{\left|Q^{+}\right|^{4}-\left|Q^{-}\right|^{4}}{\left|Q^{+}\right|^{4}+\left|Q^{-}\right|^{4}},
$$

while the unequal-CP asymmetry $B(+-,-+, \Delta t)$ is given by (122) now with $W=\frac{2-\left(\frac{p}{q}+\frac{q}{p}\right)}{2+\left(\frac{p}{q}+\frac{q}{p}\right)}$.

If CPT is conserved directly, then $X^{ \pm}$is purely imaginary, all the above results under other additional conditions are still valid, respectively, under the constraint that $X^{ \pm}$is purely imaginary.

\section{SUMMARY}

In this paper, we have studied the joint decays of a pair of two pseudoscalar neutral mesons in an entangled state of $C=-1$, as produced in $\phi$ and $B$ factories. We exactly calculated the rates of the joint decays into flavor eigenstates, taking into account direct $\mathrm{CP}$ violation and violation of $\Delta \mathcal{F}=\Delta Q$ rule. We obtained some exact results on how to extract information on $\mathrm{CP}$ and $\mathrm{CPT}$ violations from various asymmetries of the joint decays to flavor eigenstates, or joint decays to CP eigenstates. Measurement of such joint rates and asymmetries can be used to determine various parameters, including those of $\mathrm{CP}$ and CPT violations.

Remarkably, the special property of the entanglement of the $C=-1$ pair allows us to propose the meaningful and useful definition of the transition amplitude between a CP eigenstate of the meson and the decay product which is a CP eigenstate.

The equal-flavor asymmetry $A(++,--, \Delta t)$ and the equal-CP asymmetry $B(++,--, \Delta t)$ are both always independent of $\Delta t$, while the unequal-flavor asymmetry $A(+-,-+, \Delta t)$ and the unequal-CP asymmetry $B(+-,-+, \Delta t)$ generically depend on $\Delta t$.

We have considered various cases of possible direct or indirect CP or CPT violation, and obtain exact expressions of various asymmetries in various cases. Some of these exact results lead to simple yet powerful conclusions stated as several theorems.

If the equal-flavor asymmetry $A(++,--, \Delta t) \neq 0$, then we have one or two of the following violations: $(1) \mathrm{CP}$ is violated indirectly, (2) both $\mathrm{CP}$ and $\mathrm{CPT}$ are violated directly. Therefore if one assumes direct $C P T$ conservation, $A(++,--, \Delta t) \neq 0$ implies that $\mathrm{CP}$ must be violated indirectly.

Moreover, if $A(++,--, \Delta t) \neq 0$ while CPT is assumed to be conserved both directly and indirectly, then in addition to indirect $\mathrm{CP}$ violation, we can draw the following conclusions: (1) T must also be violated indirectly; (2) $\left|\left\langle l^{+}|\mathcal{H}| \bar{M}^{0}\right\rangle\right|=\left|\left\langle l^{-}|\mathcal{H}| M^{0}\right\rangle \neq\right|\left\langle l^{+}|\mathcal{H}| M^{0}\right\rangle|=|\left\langle l^{-}|\mathcal{H}| \bar{M}^{0}\right\rangle \mid$.

If the unequal-flavor asymmetry $A(+-,-+, \Delta t) \neq 0$, then $\mathrm{CP}$ must be violated directly or indirectly.

Moreover, if $A(+-,-+, \Delta t) \neq 0$ for $\Delta t \neq 0$ while CPT is assumed to be conserved both directly and indirectly, then we can draw the following conclusions: (1) $\left|\left\langle l^{+}|\mathcal{H}| \bar{M}^{0}\right\rangle\right|=\left|\left\langle l^{-}|\mathcal{H}| M^{0}\right\rangle \neq\right|\left\langle l^{+}|\mathcal{H}| M^{0}\right\rangle|=|\left\langle l^{-}|\mathcal{H}| \bar{M}^{0}\right\rangle \mid ;(2)$ $\left\langle l^{-}|\mathcal{H}| M^{0}\right\rangle=\left\langle l^{+}|\mathcal{H}| \bar{M}^{0}\right\rangle^{*} \neq 0$, which means $\Delta \mathcal{F}=\Delta Q$ rule must be violated.

For joint decays to $\mathrm{CP}$ eigenstates, in addition to various detailed results of the asymmetries, we have the following conclusions. The equal-time equal-CP rates $I\left[h^{+}, t_{a} ; h^{+}, t_{a}\right]$ and $I\left[h^{-}, t_{a} ; h^{-}, t_{a}\right]$ vanish, no matter whether $\mathrm{CP}$ or $\mathrm{CPT}$ is violated. At equal times $\Delta t=0$, the unequal-CP asymmetry $B(+-,-+, 0)$ vanishes, no matter whether $\mathrm{CP}$ or CPT is violated. On the other hand, if any equal-CP decay rate is nonzero, then $\mathrm{CP}$ must be violated.

The detailed expressions of the rates and symmetries can be used to determine the CP and CPT violating parameters. Clearly, these results are consequences of the well-known antisymmetry and entanglement of the state $\left|\Psi_{-}\right\rangle$. We hope these exact results are useful in studies on $\mathrm{CP}$ and $\mathrm{CPT}$ violations.

I am grateful to A. Di Domenico and other members of KLOE2, as well as Z. Huang, for useful discussions. This work was supported by the National Science Foundation of China (Grant No. 10875028).

[1] D. Colladay and V. A. Kostelecký, Phys. Rev. D 55, 6760 (1997). V. A. Kostelecký, Phys. Rev. Lett. 80, 1818 (1998); D. Colladay and V. A. Kostelecký, Phys. Rev. D 58, 116002 (1998); V. A. Kostelecký, Phys. Rev. D 82, 101702 (2010).

[2] E. Einstein, B. Podolsky and N. Rosen, Phys. Rev. 47, 777 (1935).

[3] M. Goldhaber, T. D. Lee and C. N. Yang, Phys. Rev. 112, 1796 (1958); D. R. Inglis, Rev. Mod. Phys. 33, 1 (1961); T. B. Day, Phys. Rev. 121, 1204 (1961); H. J. Lipkin, ibid 176, 1715 (1968). 
[4] A. Afriat and F. Selleri, The Einstein, Podolsky and Rosen Paradox in Atomic, Nuclear and Particle Physics (Plenum Press, New York, 1998); A. Bramon, R. Escribano, G. Garbarino, Found. Phys. 36, 563 (2006); R. A. Bertlmann, Lecture Notes in Physics 689 (Springer, Berlin, 2006), pp. 1-45.

[5] A. Apostolakis et al. (CPLEAR collaboration), Phys. Lett. B 422, 339 (1998).

[6] A. Go et al. (BELLE Collaboration), Phys. Rev. Lett. 99, 131802 (2007).

[7] Y. Shi, Phys. Lett. B 641, 75 (2006); Y. Shi, Phys. Lett. B 641, 492 (2006); Y. Shi and Y. L. Wu, Eur. Phys. J. C 55, 477 (2008).

[8] A. Di Domenico (KLOE Collaboration), Found. Phys. 40, 852 (2010).

[9] F. Ambrosino et al. (KLOE Collaboration), Phys. Lett. B 642, 315 (2006); G. Amelino-Camelia et al., Europhys. J. C 68, 619 (2010).

[10] B. Aubert et al. (BABAR Collaboration), Phys. Rev. Lett. 88, 221802 (2002); B. Aubert et al. (BABAR Collaboration), Phys. Rev. Lett. 89, 201802 (2002); B. Aubert et al. (BABAR Collaboration), Phys. Rev. D 70, 012007 (2004); B.Aubert et al. (BABAR Collaboration), Phys. Rev. Lett. 96, 251802 (2006); J. P. Lees et al. (BABAR Collaboration), arXiv:1207.5832,

[11] K. Abe et al. (Belle Collaboration), Phys. Rev. D 66, 071102(R) (2002); R. Louvot et al. (Belle Collaboration), Phys. Rev. Lett. 102, 021801 (2009).

[12] J. Bernabéu, F. J. Botella and J. Roldán, Phys. Lett. B 211, 226 (1980).

[13] I. Dunietz, J. Hauser and J. L. Rosner, Phys. Rev. D 35, 2166 (1987).

[14] C. D. Buchanan et al., Phys. Rev. D 45, 4088 (1992).

[15] G. D'Ambrosio, G. Isidori and A. Pugliese, arXiv:hep-ph/9411389, in L. Maiani, G. Pancheri and N. Paver. (eds.), The Second DAФNE Physics Handbook, (SIS-Publicazioni, Frascati, 1995).

[16] M. Kobayashi, A.I. Sanda, Phys. Rev. Lett. 69, 3139 (1992); Z. Xing, Phys. Rev. D 50, 2957 (1994); V.A. Kostelecky, R. Van Kooten, Phys. Rev. D 54, 5585 (1996); P. Colangelo, G. Corcella, Eur. Phys. J. C 1, 515 (1998).

[17] V. A. Kostelecký, Phys. Rev. D 61, 016002 (1999); V. A. Kostelecký, Phys. Rev. D 64, 076001 (2001).

[18] M. C. Bañuls and J. Bernabéu, Phys. Lett. 464, 117 (1999); M. C. Bañuls and J. Bernabéu, Nucl. Phys. B 590, 19 (2000); J. Bernabéu, F. Martinez-Vidal, P. Villanueva-Perez, JHEP 1208, 064 (2012).

[19] K. R. S. Balaji, W. Horn and E. A. Paschos, Phys. Rev. D 68, 076004 (2003).

[20] J. Bernabéu, N. E. Mavromatos, and J. Papavassiliou, Phys. Rev. Lett. 92, 131601 (2004); E. Álvarez and J. Bernabéu, JHEP 11, 087 (2006); N. E. Mavromatos, Found. Phys. 40, 917 (2010).

[21] A. F. Falk and A. A. Petrov, Phys. Rev. Lett. 85, 252 (2000); D. Atwood and A. A. Petrov, Phys. Rev. Lett. 71, 054032 (2005).

[22] I. Bigi and A. Sanda, CP violation, (Cambridge University Press, Cambrdge, 2009).

[23] D. Atwood and A. Soni, Phys. Rev. D 82, 036003 (2010).

[24] O. Kittle and A. Pilaftsis, Nucl. Phys. B 856, 682 (2012).

[25] Z. Huang and Y. Shi, Euro. Phys. J. C 72, 1900 (2012).

[26] Y. Shi, Euro. Phys. J. C 72, 1907(2012).

[27] K. C. Chou, W. F. Palmer, E. A. Paschos and Y. L. Wu, Eur. Phys. J. C 16, 279 (2000).

[28] L. Maiani, in L. Maiani, G. Pancheri and N. Paver. (eds.), The Second DA $\Phi$ E Physics Handbook, (SIS-Publicazioni, Frascati, 1995). 